\title{
Importancia de la velocidad y la densidad de PSA en la predicción de cáncer de próstata en la pieza de RTU o adenomectomía de pacientes con biopsia prostática previa negativa
}

\author{
Rodríguez Alonso A, González Blanco A, Barbagelata López A, Pita Fernández S*, \\ Suárez Pascual G, Bonelli Martín C, Cuerpo Pérez MA. \\ Servicio de Urología, Hospital Arquitecto Marcide. Ferrol, La Coruña. \\ *Unidad de Epidemiología Clínica y Bioestadística, Hospital Juan Canalejo. La Coruña.
}

Actas Urol Esp. 2008;32(8):779-786

\begin{abstract}
RESUMEN
IMPORTANCIA DE LA VELOCIDAD Y LA DENSIDAD DE PSA EN LA PREDICCIÓN DE CÁNCER DE PRÓSTATA EN LA PIEZA DE RTU O ADENOMECTOMÍA DE PACIENTES CON BIOPSIA PROSTÁTICA PREVIA NEGATIVA

Objetivo: Determinar variables relacionadas con el hallazgo de cáncer de próstata (CaP) en pacientes sometidos a cirugía prostática tras al menos una biopsia prostática (BP) negativa.

Material y métodos: Estudio retrospectivo de 170 pacientes sometidos a resección transuretral de próstata (RTUP) o adenomectomía entre 1999 y 2007, después de una o más BPs negativas. Se realizó un análisis multivariado de regresión logística para identificar variables relacionadas con la presencia de CaP. La capacidad predictiva de PSA, PSA densidad y PSA velocidad se determinó mediante curvas ROC y su área bajo la curva $(\mathrm{ABC})$. Se analizó la sensibilidad, especificidad y valores predictivos para diversos puntos de corte de PSA densidad y PSA velocidad.

Resultados: Se realizó adenomectomía en 104 pacientes $(61,18 \%)$ y RTUP en $66(38,82 \%)$. Se detectó CaP en la pieza quirúrgica de 16 pacientes $(9,41 \%)$. Las variables asociadas al hallazgo de $\mathrm{CaP}$ en la pieza quirúrgica fueron PSA densidad (OR:1,47;95\%IC:1,22-6,64;p:0,007) y PSA velocidad (OR:2,87;95\%IC:1,60-5,12;p<0,001). Las ABC ROC fueron 0,746, 0,793 y 0,832 , para PSA, PSA densidad y PSA velocidad, respectivamente. Los puntos de corte de PSA densidad y PSA velocidad con mayor sensibilidad y especificidad en la detección de CaP fueron 0,15 y $1 \mathrm{ng} / \mathrm{ml} /$ año, respectivamente. Los pacientes sin CaP mostraron una reducción mediana de PSA de 9,35 ng/ml $(-2,40-35,40)$, después de la cirugía.

Conclusiones: El PSA densidad y el PSA velocidad en particular, permiten predecir la presencia de CaP en la pieza de RTUP o adenomectomía, de pacientes con BPs previas negativas. La RTUP diagnóstica podría ser recomendada en pacientes con sospecha clínica de CaP, susceptibles de tratamiento curativo, con PSA velocidad $>1 \mathrm{ng} / \mathrm{ml} /$ año y una o más biopsias de saturación previas negativas.

Palabras clave: Neoplasia de próstata. Biopsia. Resección transuretral de próstata. Antígeno prostático específico. Diagnóstico.
\end{abstract}

\section{ABSTRACT}

IMPORTANCE OF PSA VELOCITY AND PSA DENSITY IN THE PREDICTION OF PROSTATE CANCER IN TURP OR OPEN PROSTATECTOMY SPECIMEN OF PATIENTS WITH PREVIOUS NEGATIVE PROSTATE BIOPSY

Purpose: To determine variables related to the finding of prostate cancer (PC) in patients who underwent surgery following at least one negative prostate biopsy (PB).

Materials and methods: A retrospective study of 170 patients who underwent transurethral resection of the prostate (TURP) or open prostatectomy between 1999 and 2007, following one or more negative PB sets. A multivariate logistic regression analysis was carried out in order to determine variables related to the finding of PC. The predictive capacity of PSA, PSA-density and PSA-velocity was assessed by means of ROC curves and the area under the curve (AUC). Sensitivity, specificity and predictive values were determined for several PSA-density and PSA-velocity cut-off points.

Results: Open prostatectomy was carried out on 104 patients $(61.18 \%)$ and TURP on $66(38.82 \%)$. PC was detected in the surgical specimen of 16 patients (9.41\%). Variables associated with the finding of PC in the surgical specimen were PSA-density (OR:1.47;95\%CI:1.22-6.64; p:0.007) and PSA-velocity (OR:2.87;95\%CI:1.60-5.12; p<0.001). The AUCs were 0.746, 0.793 and 0.832 , for PSA, PSA-density and PSA-velocity, respectively. The most sensitive PSA-density and PSA-velocity cut-off points in detecting PC were 0.15 and $1 \mathrm{ng} / \mathrm{ml} /$ year, respectively. Patients without PC showed a median PSA reduction of $9.35 \mathrm{ng} / \mathrm{ml}(-2.40$ -35.40 ), following surgery.

Conclusions: PSA-density and PSA-velocity in particular, allow for the prediction of the presence of PC in the TURP or open prostatectomy specimen of patients with previously negative PBs. Diagnostic TURP could prove useful in patients with clinical suspicion of PC, susceptible to curative treatment, with PSA-velocity $>1 \mathrm{ng} / \mathrm{ml} /$ year and one or more negative saturation biopsies.

Keywords: Prostatic neoplasms. Biopsy. Transurethral resection of prostate. Prostate-specific antigen. Diagnosis. 
$\mathrm{L}^{\mathrm{a}}$ a elevada tasa de falsos negativos de la biopsia de próstata (BP), conduce en la mayoría de los casos de biopsia negativa, a una segunda biopsia, salvo que se normalice el PSA ${ }^{1}$.

La probabilidad de diagnosticar cáncer de próstata $(\mathrm{CaP})$ a partir de la segunda biopsia es reduci$\mathrm{da}^{2}$, sin embargo la posibilidad de falsos negativos sigue existiendo independientemente del número de biopsias efectuadas, ya que el diagnóstico de certeza sólo podría establecerse con la pieza quirúrgica. Aún así, en muchos casos parece razonable cesar en la realización de BPs y monitorizar el PSA, considerando que su elevación es de naturaleza benigna.

La zona periférica de la próstata es fácilmente accesible al tacto rectal, y a la aguja de biopsia, sin embargo la zona transicional, donde se ubican el 20\% de los tumores, no puede ser muestreada con la misma precisión, especialmente si el volumen prostático es elevado, ya que la mayoría de los CaP de la zona transicional tienen una localización anterior ${ }^{3,4}$.

Según diversos autores, la probabilidad de encontrar CaP en la pieza de resección transuretral de próstata (RTUP) de pacientes con BPs negativas previas es del 4-28\%2. Por ello sería importante encontrar parámetros que determinasen qué pacientes con BPs negativas tienen mayor probabilidad de padecer $\mathrm{CaP}$, para emplear todas las estrategias diagnósticas disponibles. La RTUP es probablemente la mejor, ya que permite obtener muestras histológicas de áreas prostáticas difícilmente accesibles a la BP.

El propósito de este estudio es determinar qué variables influyen en el hallazgo de CaP en la pieza de RTUP o adenomectomía, de un grupo de pacientes con uno o más sets de BPs negativas. Secundariamente se pretende definir el papel de la RTUP en el diagnóstico del CaP tras múltiples BPs negativas.

\section{MATERIAL Y MÉTODOS}

Se realizó un estudio observacional de cohorte retrospectivo, analizando 170 pacientes tratados con RTUP o adenomectomía entre 1999 y 2007 en el Hospital Arquitecto Marcide, Área Sanitaria de Ferrol, La Coruña.

A todos los pacientes se les practicó al menos una $\mathrm{BP}$, con resultado negativo. Los criterios de $\mathrm{BP}$ fueron los siguientes: tacto rectal sospechoso, o PSA $>10 \mathrm{ng} / \mathrm{ml}$, o PSA entre 4 y $10 \mathrm{ng} / \mathrm{ml}$ con un cocien- te PSA libre/total $<20 \%$, o PSA densidad (PSAD) $>0,15$. La BP fue realizada utilizando un ecógrafo BK Leopard 2001, con un transductor transrectal biplanar de 7,5 Mhz y una aguja de 18G.

Se realizó una BP transrectal ecodirigida de 6 cilindros hasta diciembre de 2001, y a partir de entonces se siguió un esquema de 10 cilindros (6 muestras parasagitales y 4 pósterolaterales).

Se realizó biopsia de saturación de 24 cilindros, tomando 20 muestras periféricas y 4 transicionales anteriores, siguiendo un esquema e indicación previamente publicados ${ }^{5}$.

Todos los pacientes fueron valorados preoperatoriamente mediante anamnesis, exploración física, tacto rectal, PSA, PSA complex, uroflujometría, medición del volumen residual postmiccional y determinación del volumen prostático mediante ecografía transrectal (TRUS).

La indicación para realizar cirugía prostática fue el tratamiento de sintomas severos del tracto urinario inferior, refractarios al tratamiento farmacológico y/o uroflujometría con flujo máximo patológico y/o residuo postmiccional elevado, o sonda vesical permanente por retención urinaria.

El abordaje quirúrgico se decidió en función del volumen prostático determinado por TRUS, realizándose RTUP si este era $\leq 60$ cc y adenomectomía si este era >60 cc. La RTUP se realizó con corriente monopolar hasta 2004 y desde entonces con corriente bipolar.

La determinación del PSA se efectuó mediante el ensayo ADVIA Centaur ${ }^{\circledR}$ (Bayer Diagnostics, Tarrytown, New York).

EL PSA velocidad (PSAV) se calculó utilizando un método aritmético simple no restringido ${ }^{6}$, empleando dos mediciones de PSA: PSA previo a primera BP (PSA1) y PSA previo a cirugía prostática (PSA2), independientemente del tiempo transcurrido entre ambas:

(PSA2 - PSA1) $\times 365 / N$

N: Tiempo en dias transcurrido entre PSA2 y PSA1.

\section{Análisis estadístico}

Se realizó un análisis descriptivo de todas las variables. Las variables cuantitativas se expresaron como media \pm desviación típica y las variables cualitativas como valor absoluto y porcentaje, con su 95\%IC. La comparación de variables cuantitativas entre grupos se realizó mediante el test t-Student o 
el test U de Mann-Whitney, según procediese. El contraste de la normalidad se realizó con el test Kolgomorov-Smirnov. Para la comparación de variables cualitativas se empleó el estadístico $\chi^{2}$ y el cálculo del OR con su 95\% IC. Se efectuó un análisis de regresión logística, para determinar variables asociadas al hallazgo de $\mathrm{CaP}$ en la pieza quirúrgica. Se calculó la sensibilidad, especificad y valores predictivos para distintos puntos de corte de PSAD y PSAV. La capacidad del PSA, PSAD y PSAV para predecir la probabilidad de $\mathrm{CaP}$ en la pieza quirúrgica se determinó mediante las correspondientes curvas ROC y su área bajo la curva $(\mathrm{ABC})$. El análisis estadístico se efectuó mediante el programa SPSS 14.0 para Windows.

\section{RESULTADOS}

En 104 pacientes $(61,18 \%)$ se realizó adenomectomía y en $66(38,82 \%)$ RTUP.

Se detectó $\mathrm{CaP}$ en la pieza quirúrgica de 16 pacientes $(9,41 \%)$. Se detectó prostatitis crónica y/o aguda en alguna de las biopsias, en 79 pacientes $(46,47 \%)$ y en la pieza quirúrgica de 58 pacientes $(34,12 \%)$. Entre los 79 pacientes con prostatitis en alguna de las biopsias, se detectó $\mathrm{CaP}$ en 8 casos $(10,13 \%)$ y entre los 91 pacientes sin prostatitis se detectó CaP en 8 casos $(8,79 \%)$, no siendo las diferencias significativas $(\mathrm{p}=0,766)$.

La Tabla 1 muestra las características clínicas de los pacientes de la muestra estudiada.
El número de biopsias por paciente fue el siguiente: 1 BP en 74 casos (43,53\%), 2 BP en 57 casos (33,53\%), 3 BP en 21 casos (12,35\%) y 4 BP en 18 casos $(10,59 \%)$.

En 21 pacientes el tacto rectal previo a la cirugía era sospechoso, presentando CaP 2 de estos pacientes $(9,52 \%)$, mientras que en 19 casos $(90,48 \%)$ no se observó malignidad en la pieza quirúrgica. Entre los 149 pacientes con tacto rectal normal, 14 (9,40\%) presentaban CaP, y en $135(90,60 \%)$ no se observó malignidad ( $\mathrm{p}=0,985)$. Las diferencias entre el grupo de pacientes sin malignidad y el grupo con CaP, se muestran en la Tablas 2 y 3.

Se realizó un análisis multivariado de regresión logística para predecir variables asociadas a la existencia de $\mathrm{CaP}$ en la pieza quirúrgica, ajustando por edad en cirugía prostática, tacto rectal, existencia de prostatitis crónica, PSA previo a cirugía, PSAD previo a cirugía, y PSAV. Se observó que las variables PSAV (OR: 2,87; p: <0,0001) y PSAD (OR: 1,47; p: 0,007) predecían de forma independiente la existencia de CaP en la pieza quirúrgica (Tabla 4).

La capacidad predictiva de PSA, PSAD y PSAV se determinó mediante curvas ROC y sus correspondientes ABC. Las ABC eran 0,746, 0,793 y 0,832, para el PSA, PSAD y PSAV, respectivamente (Fig. 1).

Se determinó la validez (sensibilidad y especificidad) y la seguridad (valor predictivo positivo y valor predictivo negativo) del PSAV y PSAD, para diferentes puntos de corte. Las diferencias entre los grupos formados se exponen en las Tablas 5 y 6 .

Tabla 1. Características clínicas de la población del estudio.

\begin{tabular}{|c|c|c|c|c|}
\hline Variable & Media & Mediana & DT & Mín-Máx \\
\hline Edad en primera $\mathrm{BP}$ & 67,96 & 67,62 & 7,16 & $52-85$ \\
\hline Edad en cirugía & 70,01 & 70,11 & 6,75 & $52-86$ \\
\hline TRUS en $1^{\mathrm{a} B P}$ & 89,36 & 85,10 & 38,06 & $26,00-245,00$ \\
\hline PSA $1^{\mathrm{a} B P}$ & 12,53 & 10,65 & 7,91 & $3,10-43,20$ \\
\hline PSA complex $1^{\mathrm{a} B P}$ & 8,69 & 7,35 & 4,85 & $2,50-29,08$ \\
\hline Cociente PSAl/t $1^{\mathrm{a}} \mathrm{BP}$ & 18,53 & 18,15 & 7,57 & $6,30-43,10$ \\
\hline PSA densidad $1^{a} B P$ & 0,15 & 0,12 & 0,09 & $0,03-0,48$ \\
\hline TRUS en cirugía & 89,57 & 85,40 & 38,08 & $26,00-245,00$ \\
\hline PSA cirugía & 13,17 & 11,10 & 8,05 & $3,00-43,20$ \\
\hline PSA complex cirugía & 10,40 & 9,70 & 5,89 & $2,60-31,39$ \\
\hline Cociente PSAl/t cirugía & 20,43 & 19,00 & 8,44 & $6,30-46,80$ \\
\hline PSA densidad cirugía & 0,17 & 0,13 & 0,13 & $0,03-0,77$ \\
\hline Número de BPE previas & 1,90 & 2 & 1,54 & $1-4$ \\
\hline Número de cilindros previos & 20,42 & 16 & 19,68 & 6-68 \\
\hline Tiempo entre $1^{\mathrm{a}} \mathrm{BP}$ y cirugía (meses) & 24,95 & 15,31 & 19,33 & $0,46-92,11$ \\
\hline Tiempo entre última BP y cirugía (meses) & 15,10 & 14,70 & 8,33 & $0,46-68,00$ \\
\hline
\end{tabular}

DT: Desviación Típica. Mín-Máx: Mínimo-Máximo. 
Tabla 2. Diferencias encontradas entre los grupos de no malignidad y cáncer de próstata.

\begin{tabular}{|c|c|c|c|c|c|}
\hline \multirow[t]{2}{*}{ Variable } & \multicolumn{2}{|c|}{ No malignidad } & \multicolumn{2}{|c|}{ Cáncer de próstata } & \multirow{2}{*}{$\begin{array}{l}\text { Valor } \\
\text { de } p\end{array}$} \\
\hline & Media \pm DT & Mediana & Media \pm DT & Mediana & \\
\hline Edad $1^{\mathrm{a}} \mathrm{BP}$ & $67,77 \pm 7,21$ & 66,87 & $69,85 \pm 6,67$ & 70,52 & $0,270^{\mathrm{a}}$ \\
\hline Edad cirugía & $69,73 \pm 6,83$ & 69,92 & $72,74 \pm 5,43$ & 73,76 & $0,090^{\mathrm{a}}$ \\
\hline TRUS $1^{\mathrm{a}} \mathrm{BP}$ & $91,57 \pm 37,19$ & 85,40 & $68,68 \pm 41,15$ & 50,25 & $0,020^{\mathrm{b}}$ \\
\hline PSA $1^{\mathrm{a}} \mathrm{BP}$ & $12,63 \pm 8,27$ & 10,55 & $11,58 \pm 2,89$ & 10,65 & $0,443^{\mathrm{b}}$ \\
\hline PSA complex $1^{\mathrm{a}} \mathrm{BP}$ & $8,32 \pm 4,98$ & 7,09 & $11,14 \pm 3,14$ & 10,72 & $0,010^{\mathrm{b}}$ \\
\hline $\mathrm{PSA} 1 / \mathrm{t} 1^{\mathrm{a}} \mathrm{BPE}$ & $19,82 \pm 7,21$ & 19,00 & $9,80 \pm 2,23$ & 10,20 & $0,000^{\mathrm{a}}$ \\
\hline PSA densidad $1^{a} \mathrm{BP}$ & $0,14 \pm 0,09$ & 0,12 & $0,22 \pm 0,11$ & 0,25 & $0,009^{b}$ \\
\hline TRUS cirugía & $94,80 \pm 37,33$ & 88,45 & $70,32 \pm 43,71$ & 52,10 & $0,023^{\mathrm{b}}$ \\
\hline PSA cirugía & $12,63 \pm 7,89$ & 11,10 & $18,42 \pm 7,89$ & 17,75 & $0,001^{\mathrm{b}}$ \\
\hline PSA complex cirugía & $9,02 \pm 4,29$ & 8,40 & $16,12 \pm 6,44$ & 17,80 & $0,000^{b}$ \\
\hline PSA $1 /$ t cirugía & $21,22 \pm 8,37$ & 20,90 & $14,98 \pm 7,04$ & 12,30 & $0,028^{a}$ \\
\hline PSA densidad cirugía & $0,15 \pm 0,09$ & 0,12 & $0,38 \pm 0,24$ & 0,33 & $0,000^{\mathrm{b}}$ \\
\hline PSA velocidad & $0,28 \pm 0,72$ & 0,00 & $1,33 \pm 1,49$ & 0,77 & $0,000^{b}$ \\
\hline Número de BP & $1,82 \pm 1,12$ & 1,00 & $2,75 \pm 1,77$ & 2,00 & $0,013^{b}$ \\
\hline Número total de cilindros & $18,78 \pm 14,87$ & 16,00 & $36,25 \pm 24,79$ & 34,00 & $0,011^{\mathrm{b}}$ \\
\hline
\end{tabular}

a: Test U Mann-Whitney. ${ }^{\text {b: }}$ Test T Student.

Tabla 3

\begin{tabular}{lccc}
\hline Variable & Odds Ratio & $\mathbf{9 5 \% I C}$ & Valor de p \\
\hline $\begin{array}{l}\text { Tacto rectal } \\
\text { sospechoso (no/sí) }\end{array}$ & 1,01 & $0,21-4,81$ & $0,985^{\mathrm{a}}$ \\
$\begin{array}{l}\text { Prostatitis } \\
\text { crónica (no/sí) }\end{array}$ & 1,16 & $0,41-3,27$ & $0,766^{\mathrm{a}}$ \\
\hline
\end{tabular}

a: Estadístico chi-cuadrado $\left(\chi^{2}\right)$

Tabla 4. Modelo multivariado de regresión logística para predecir variables relacionadas con el hallazgo de cáncer de próstata en la pieza quirúrgica.

\begin{tabular}{lccc}
\hline Variable & Odds Ratio & $\mathbf{9 5 \%} \mathbf{I C}$ & $\begin{array}{c}\text { Valor } \\
\mathbf{d e ~} \mathbf{p}\end{array}$ \\
\hline Edad & 1,10 & $0,99-1,22$ & 0,056 \\
$\begin{array}{l}\text { Tacto rectal sospechoso } \\
\text { (no/sí) }\end{array}$ & 1,01 & $0,15-6,44$ & 0,991 \\
$\begin{array}{l}\text { Prostatitis crónica } \\
\text { (no/sí) }\end{array}$ & 0,76 & $0,21-2,76$ & 0,681 \\
PSA & 0,96 & $0,88-1,06$ & 0,519 \\
PSA densidad & 1,47 & $1,22-6,64$ & 0,007 \\
PSA velocidad & 2,87 & $1,60-5,12$ & 0,000 \\
\hline
\end{tabular}

En los pacientes sin CaP, el primer PSA postoperatorio fue $2,40 \pm 2,81 \mathrm{ng} / \mathrm{ml}(0,10-11,60)$, con una mediana de $1,20 \mathrm{ng} / \mathrm{ml}$. La reducción media de PSA tras la cirugía prostática fue de $11,49 \pm 8,56 \mathrm{ng} / \mathrm{ml}$
$(-2,40-35,40)$, siendo la reducción mediana de 9,35 $\mathrm{ng} / \mathrm{ml}$, en los pacientes sin malignidad.

Las características histológicas de los CaP hallados en la pieza quirúrgica y el tratamiento efectuado se recogen en la Tabla 7 .

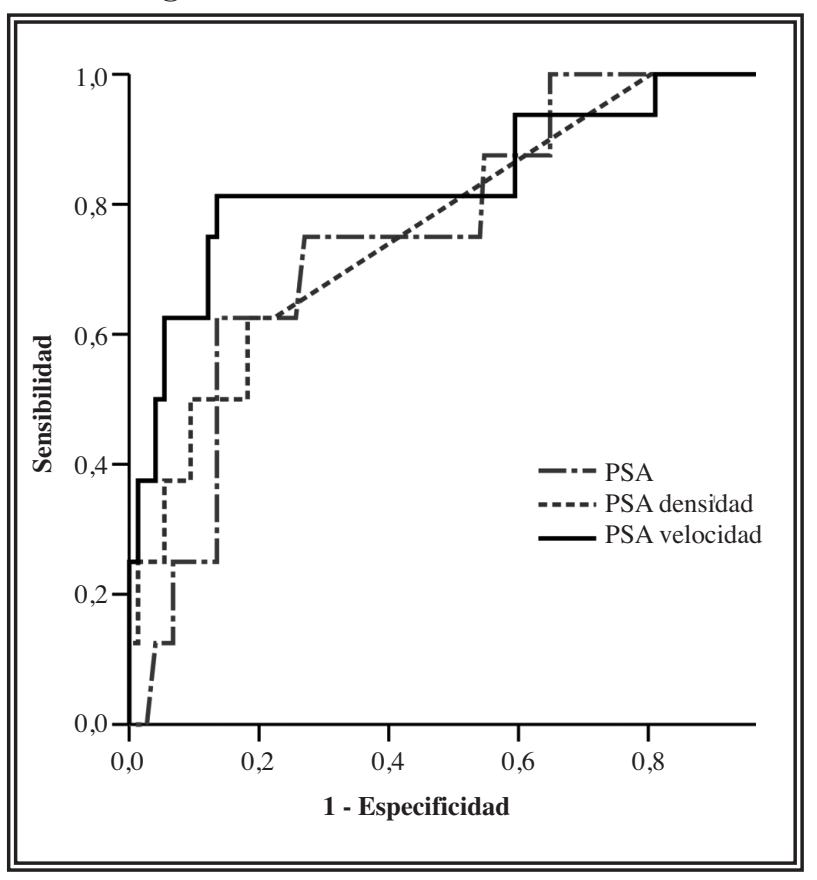

FIGURA 1. Curvas ROC: Las áreas bajo las curvas ROC fueron 0,746 (0,641-0,869) para el PSA, 0,793 (0,659$0,904)$ para el PSA densidad y $0,832(0,713-0,963)$ para el PSA velocidad. 
Tabla 5. Sensibilidad, especificidad y valores predictivos para diversos puntos de corte de PSA velocidad.

\begin{tabular}{lcccccc}
\hline \multicolumn{7}{c}{ PSA velocidad (ng/ml/año) } \\
\hline Cutoff & Sensibilidad & Especificidad & VPP & VPN & Odds Ratio (95\%IC) & Valor de $\mathbf{p}$ \\
\hline 0,75 & 50,00 & 86,84 & 28,57 & 94,28 & $6,60(2,22-19,57)$ & $<0,001$ \\
1 & 50,00 & 88,81 & 32,00 & 94,40 & $7,94(2,63-23,90)$ & $<0,001$ \\
1,5 & 37,50 & 90,79 & 30,00 & 93,24 & $5,91(1,86-18,71)$ & 0,001 \\
2 & 37,50 & 92,10 & 33,33 & 93,33 & $7,00(2,17-22,58)$ & $<0,001$ \\
3 & 25,00 & 98,68 & 66,66 & 92,59 & $25,00(4,14-150,68)$ & $<0,001$ \\
\hline
\end{tabular}

a: Estadístico chi-cuadrado $\left(\chi^{2}\right)$. Cutoff: Punto de corte. VPP: Valor predictivo positivo. VPN: Valor predictivo negativo.

Tabla 6. Sensibilidad, especificidad y valores predictivos para diversos puntos de corte de PSA densidad.

\begin{tabular}{lcccccc}
\hline \multicolumn{7}{c}{ PSA densidad } \\
\hline Cutoff & Sensibilidad & Especificidad & VPP & VPN & Odds Ratio (95\%IC) & Valor de $\mathbf{p}$ \\
\hline 0,10 & 87,50 & 34,00 & 12,38 & 96,22 & $3,60(0,78-16,48)$ & 0,080 \\
0,15 & 75,00 & 60,00 & 16,66 & 95,74 & $4,50(1,38-14,61)$ & 0,007 \\
0,20 & 75,00 & 73,33 & 24,49 & 96,58 & $9,16(2,78-30,14)$ & $<0,001$ \\
0,25 & 62,50 & 89,33 & 38,46 & 95,71 & $13,95(4,47-43,52)$ & $<0,001$ \\
0,30 & 62,50 & 94,66 & 55,55 & 95,94 & $29,58(8,58-101,98)$ & $<0,001$ \\
\hline
\end{tabular}

a: Estadístico chi-cuadrado $\left(\chi^{2}\right)$. Cutoff: Punto de corte. VPP: Valor predictivo positivo. VPN: Valor predictivo negativo.

Tabla 7. Histopatología y tratamiento aplicado a los pacientes con cáncer de próstata.

\begin{tabular}{lc}
\hline Variable & $\mathbf{N}(\%)$ \\
\hline Gleason score & \\
$4(2+2)$ & $1(6,25)$ \\
$5(3+2)$ & $3(18,75)$ \\
$6(3+3)$ & $2(12,50)$ \\
$7(3+4)$ & $3(18,75)$ \\
$7(4+3)$ & $4(25,00)$ \\
$8(4+4)$ & $3(18,75)$ \\
\% Tumor en la pieza & \\
$<5 \%$ & $2(12,50)$ \\
$>5 \%$ & $14(87,50)$ \\
Tratamiento efectuado & \\
Vigilancia expectante & $4(25,00)^{1}$ \\
Radioterapia externa & $8(50,00)$ \\
Prostatectomia radical & $4(50,00)$ \\
\hline
\end{tabular}

1: Dos pacientes recibieron tratamiento posterior

(Hormonoterapia y Radioterapia externa).

El seguimiento medio de los pacientes con CaP fue 33,61 meses $(9,11-83,02)$, con una mediana de 24,67 meses. Durante este intervalo se produjeron 4 muertes, y sólo una de ellas a consecuencia del $\mathrm{CaP}$.

En 11 pacientes se realizó una nueva biopsia tras la cirugía prostática, en 6 casos por persisten- cia de PSA elevado y en 5 por elevación de PSA, tras una reducción postoperatoria. En 6 casos la biopsia prostática demostró CaP. En total 22 de los 170 pacientes $(12,94 \%)$ presentaron $\mathrm{CaP}$, bien en la pieza quirúrgica o bien en una BP posterior.

\section{DISCUSIÓN}

Las herramientas empleadas para diferenciar patología prostática benigna y CaP presentan una precisión menor de lo deseable. La sensibilidad del tacto rectal es reducida, ya que hasta el 50\% de los pacientes se diagnostican en estadio Tlc. El PSA presenta una baja especificidad, ya que se trata de una molécula órgano-específica y no cáncer-específica y su elevación puede ser ocasionada por otros procesos distintos al $\mathrm{CaP}^{1,7}$.

Otro problema es que la tasa de falsos negativos de la BP es del 14-35\% $\%^{1,3}$. A consecuencia de la baja sensibilidad de la BP convencional, la probabilidad de realización de una segunda biopsia es elevada (13-41\%), alcanzando el 60\% en caso de PIN ${ }^{5}$. En la segunda BP, la tasa de detección de CaP es del 10$19 \%$, siendo habitualmente inferior al $10 \%$ en la tercera biopsia y sucesivas ${ }^{1,6}$.

Por estos motivos, la tendencia actual es obtener un mayor número de cilindros ${ }^{8}$, realizando biopsias 
ampliadas desde la primera BP y biopsias de saturación, que algunos grupos realizan a partir de la segunda biopsia, incrementando hasta un 13,540\% la detección de $\mathrm{CaP}^{3,5,9}$. En estos esquemas de biopsia de saturación además de incrementar el número de cilindros totales, se biopsian áreas prostáticas que son asiento frecuente de tumores y que habitualmente no se biopsian, como la zona periférica más pósterolateral y la zona transicional anterior.

A pesar de la repetición de biopsias, de incrementar el número de cilindros y de seleccionar los lugares de punción, la BP continúa presentando falsos negativos. En este punto surge un dilema frecuente hoy en día: ¿qué hacer en aquellos pacientes con varias BP negativas y persistencia de criterios clínicos de biopsia?.

Este dilema es especialmente importante en los pacientes más jóvenes, en los que el hallazgo de CaP permitiría realizar un tratamiento potencialmente curativo $^{4}$. En este grupo de pacientes es necesario definir métodos diagnósticos útiles para diferenciar patología benigna de CaP, que nos permitan decidir si seguimos tomando muestras de tejido prostático o bien es razonable un seguimiento expectante. En los pacientes de mayor edad, la importancia de realizar un diagnóstico precoz es relativa, ya que la eventual existencia de un CaP no diagnosticado en dos o más sets de biopsias, probablemente no comprometerá la supervivencia del paciente, ya que suele tratarse de CaP con baja significación clínica ${ }^{10}$.

Los pacientes sintomáticos pueden ser sometidos a cirugía prostática. En los pacientes asintomáticos algunos autores proponen la realización de RTUP, al observar que los pacientes con PSA elevado y múltiples biopsias negativas presentan en su mayoría datos urodinámicos de obstrucción infravesical $^{11}$

La indicación quirúrgica de todos los pacientes de nuestro estudio fue la existencia de síntomas del tracto urinario inferior. De los 16 casos de CaP, 6 pacientes tenían una biopsia de saturación y 4 pacientes 2 biopsias de saturación. El número medio de biopsias previas a la cirugía en los pacientes con CaP fue 2,75 y el número medio de cilindros tomados de 36,25.

Los falsos negativos de la BP pueden corresponder a tumores localizados en áreas de la zona periférica no muestreadas o bien en la zona transicional.
Todos los tumores en nuestra serie estaban obviamente ubicados en la zona transicional de la próstata y la mediana de PSA de los pacientes con CaP fue 17,75. A pesar de esta cifra de PSA, estos pacientes podrian ser susceptibles de tratamiento curativo, ya que los tumores de la zona transicional suelen ser mejor diferenciados, con menor probabilidad de metástasis y mayor supervivencia libre de recidiva bioquímica que los tumores periféricos. Asimismo los tumores transicionales presentan una elevada probabilidad de organoconfinación y curación aunque el PSA sea $>20 \mathrm{ng} / \mathrm{ml}^{4,12}$.

Nuestro estudio revela que las variables con capacidad para predecir de forma independiente la presencia de CaP son PSAD y PSAV. El volumen ecográfico medio de la próstata fue de 94,80 cc en pacientes sin evidencia de malignidad y de 70,32 cc en caso de CaP ( $p=0,023)$. El valor medio de PSAD fue 0,15 y 0,38, en pacientes sin malignidad y con $\mathrm{CaP}$, respectivamente $(\mathrm{p}<0,001)$. Estos hallazgos son coincidentes con los de otros autores que observan que el volumen prostático y el PSAD, resultan de gran utilidad en la detección precoz de $\mathrm{CaP}^{13}$.

En nuestra serie se detectó $\mathrm{CaP}$ en la pieza quirúrgica en el 9,41\% de los casos. En concordancia con los hallazgos de otros autores ${ }^{1,2,11,14}$, la mayoría de los CaP $(81,25 \%)$ de nuestra serie fueron clínicamente significativos, basándose en su Gleason, PSA, PSAD y categoría $\mathrm{T}(87,50 \% \mathrm{~T} 1 \mathrm{~b})$.

De los resultados de nuestro estudio se desprende que los pacientes con una o más BP negativas tienen una mayor probabilidad de padecer un CaP cuando el PSAD y sobre todo el PSAV son elevados. En este grupo de pacientes deberían emplearse todas las estrategias diagnósticas disponibles en la actualidad: biopsia de saturación, biopsia de zona transicional anterior, RTUP diagnóstica, RM y espectroscopia basada en $\mathrm{RM}^{4}$.

La biopsia de saturación con muestreo de la zona de transición es la primera opción lógica a realizar en pacientes con varias biopsias previas negativas, porque el incremento del número de cilindros biópsicos se asocia a un incremento en la detección de CaP y porque permitiría detectar tumores limitados a la zona transicional ${ }^{4}$. Sin embargo, la rentabilidad diagnóstica de la biopsia de la zona transicional ha sido cuestionada, al observar una reducida frecuencia de tumores que afectan exclusivamente a la zona de transición. ${ }^{15}$ 
La RM y la espectroscopia basada en RM, en el diagnóstico de CaP, está limitada en algunos centros por razones económicas, por otra parte, su aplicación práctica para dirigir las biopsias transrectales a las lesiones detectadas está actualmente bajo estudio $^{16,17}$.

Recientemente, algunos autores han sugerido realizar RTUP en pacientes con varias BP negativas y sospecha clínica de $\mathrm{CaP}$. El planteamiento de esta modalidad diagnóstica es realizar un muestreo exhaustivo de la zona transicional anterior de la próstata, que es menos accesible a la biopsia transrectal, y donde se ubican la mayoría de los tumores de la zona transicional ${ }^{4,7}$.

La oportunidad de la RTUP diagnóstica es controvertida. Algunos autores la recomiendan, como Ornstein DK et al, que detectaron CaP en un $16,5 \%$ de pacientes sintomáticos, siendo $\geq \mathrm{T} 1 \mathrm{~b}$ en el $71 \%$ de los casos, concluyendo que se debe realizar RTUP diagnóstica en pacientes que se puedan beneficiar de un diagnóstico precoz del $\mathrm{CaP}^{14}$. Puppo $\mathrm{P}$ et al. detectaron $\mathrm{CaP}$ mediante RTUP en el 50\% de los pacientes con $\mathrm{PSAV}>\mathrm{lng} / \mathrm{ml} /$ año, siendo todos los tumores clínicamente significativos. ${ }^{2}$ Niesel $\mathrm{T}$ et al detectaron CaP mediante RTUP en el 40,8\% de los pacientes con clínica obstructiva y criterios de biopsia, realizando simultáneamente RTUP y BP. ${ }^{18}$ Esta elevada tasa de detección puede ser debida a que los pacientes no habían sido biopsiados previamente.

Otros autores sólo recomiendan RTUP diagnóstica en pacientes seleccionados, así Zigeuner R et al observan una tasa global de CaP del 7,9\%, concluyendo que la incidencia es sólo ligeramente superior a la de cáncer incidental en pacientes sin biopsias previas. ${ }^{1}$ Los autores sólo recomiendan RTUP en aquellos pacientes con tacto rectal sospechoso y síntomas de vaciado, en los que la tasa de CaP era del 16,5\%.

No existen en la actualidad bases científicas claras para realizar de forma rutinaria una RTUP diagnóstica, como tampoco existe consenso sobre cuántas biopsias deben realizarse, con qué intervalo, ni a qué pacientes, ni cuando se debe parar de biopsiar ${ }^{19}$.

Para adoptar decisiones clínicas basadas en el PSAD y PSAV, es necesario definir un punto de corte en ambos valores. Nuestra opinión es que en los individuos mayores, con una esperanza de vida $<10$ años, es conveniente utilizar un punto de corte con el mayor valor predictivo positivo posible, ya que considerando el balance riesgo-beneficio de una
RTUP diagnóstica, esta debería practicarse cuando la probabilidad de detectar CaP sea muy elevada, realizando el menor número de RTUP innecesarias.

Por el contrario, en los pacientes más jóvenes, con una esperanza de vida >10 años, y subsidiarios de tratamiento potencialmente curativo, ${ }^{20}$ debería emplearse un punto de corte con elevada sensibilidad, para detectar el mayor número posible de pacientes con CaP, evidentemente a expensas de un mayor número de RTUP innecesarias.

Teniendo en cuenta estas premisas, creemos que en los pacientes mayores los puntos de corte óptimos de PSAD y PSAV serían 0,30 y $3 \mathrm{ng} / \mathrm{ml} / \mathrm{año}$, respectivamente, mientras que en los pacientes más jóvenes, estos deberían ser 0,15 y $1 \mathrm{ng} / \mathrm{ml} / \mathrm{año}$, respectivamente.

El PSAD es un parámetro subjetivo, ya que en la determinación del volumen ecográfico prostático existe una inevitable variabilidad intra e interobservador. Adicionalmente, un punto de corte con elevada sensibilidad se asocia a un bajo nivel de especificidad, por lo que adoptando un punto de corte de 0,15 , se habrian seleccionado preoperatoriamente 12 de los 16 pacientes con CaP, practicando 59 RTUP innecesarias. Por el contrario, el PSAV es un parámetro fácilmente reproducible, por lo que creemos que puede tener una mayor aplicación clínica. Tomando un punto de corte de PSAV de 3 $\mathrm{ng} / \mathrm{ml} / \mathrm{año}, 4$ de los 16 pacientes con CaP se habrían seleccionado preoperatoriamente, con 2 RTUP innecesarias. Con un punto de corte de PSAV de 1 ng/ml/año, 8 de 16 pacientes con CaP se habrían identificado preoperatoriamente con 17 RTUP innecesarias en los restantes 154 pacientes.

En nuestro estudio la cirugía prostática fue efectuada con fines terapéuticos y no diagnósticos. A pesar de que esto supone una limitación, nuestra opinión a la vista de los resultados es que la RTUP diagnóstica podría estar indicada en pacientes con

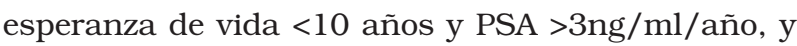
particularmente en pacientes con esperanza de vida $>10$ años y PSAV > lng/ml/año. En nuestra serie, se diagnóstico $\mathrm{CaP}$ en el 32,00\% de los pacientes con $\mathrm{PSAV}>$ lng/ml/año y en el $66,67 \%$ de los casos con

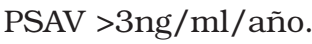

Es importante que los pacientes comprendan que la RTUP diagnóstica no es un procedimiento estándar, y que sólo se realiza con el propósito de identificar un $\mathrm{CaP}$, por ello deben conocer adecuadamente las posibles complicaciones derivadas de la técnica ${ }^{2}$. 
La RTUP cumpliría una función múltiple desde nuestro punto de vista, ya que permite establecer el diagnóstico de CaP y eventualmente aliviar los síntomas del tracto urinario inferior. Otro de los beneficios de la RTUP es su efecto reductor del PSA, que hace desaparecer en muchos pacientes la indicación de biopsia y por lo tanto la ansiedad generada por la incertidumbre. Por otra parte, al reducir considerablemente el volumen prostático, el muestreo prostático sería más preciso en una eventual biopsia posterior ${ }^{4}$. En nuestro estudio, la mediana de PSA postoperatorio de los pacientes sin evidencia de malignidad fue de $1,20 \mathrm{ng} / \mathrm{ml}$, siendo el PSA $<4$ en el $80,55 \%$ de los casos. Asimismo entre la cifra de PSA preoperatorio y el primer PSA postoperatorio se observó una reducción mediana del 9,35 ng/ml $(-2,40-35,40)$. La indicación de BP desapareció en el $83,33 \%$ de los pacientes sin evidencia de malignidad en la pieza quirúrgica.

La RTUP presenta en la actualidad una baja tasa de complicaciones importantes, que son aún más reducidas si se utiliza energía bipolar ${ }^{21}$. Por otra parte la realización de RTUP no interfiere en el eventual tratamiento de intención curativa, bien radioterapia externa, o prostatectomía radical $^{2,7}$.

\section{CONCLUSIONES}

Los resultados de nuestro estudio revelan que el PSAD y particularmente el PSAV permiten predecir la presencia de CaP en la pieza de RTUP o adenomectomía, en pacientes con BPs previas negativas.

A pesar de no existir una base científica sólida que respalde la utilización de RTUP diagnóstica, creemos que esta técnica podría ser considerada como una opción válida en pacientes con sospecha clínica de $\mathrm{CaP}$ que presentan un PSAV $>1 \mathrm{ng} / \mathrm{ml} /$ año y una o más biopsias de saturación negativas, en los cuales el diagnóstico de CaP permitiría la ejecución de un tratamiento potencialmente curativo.

\section{REFERENCIAS}

1. Zigeuner R, Schips L, Lipsky K, Auprich M, Salfellner M, Rehak P, et al Detection of prostate cancer by TURP of open surgery in patients with previously negative transrectal prostate biopsies. Urology. 2003;62(5): 883-887.

2. Puppo P, Introini C, Calvi P, Naselli A. Role of transurethral resection of the prostate and biopsy of the peripheral zone in the same session after repeated negative biopsies in the diagnosis of prostate cancer. Eur Urol. 2006;49(5):873-878

3. Borboroglu PG, Comer SW, Riffenburgh RH, Amling CL. Extensive repeat transrectal ultrasound guided prostate biopsy in patients with previous benign sextant biopsies. J Urol. 2000;163(1):158-162.
4. Bratt $O$. The difficult case in prostate cancer diagnosis when is a "Diagnostic TURP" indicated?. Eur Urol. 2006;49(5):769-771.

5. Rodríguez Alonso A, González Blanco A, Pita Fernández S, Suárez Pascual G, Bonelli Martín C, Lorenzo Franco J, et al. Prostate cancer diagnosis using 24 cores extended biopsy. Actas Urol Esp. 2005;29(10): 934-942.

6. Yu X, Han M, Loeb S, Gashti S, Yeh JT, Roehl KA, Catalona WJ. Comparison of methods for calculating prostate specific antigen velocity. J Urol. 2006;176(6 Pt 1):2427-2431.

7. Puppo P. Repeated negative prostate biopsies with persistently elevated or rising PSA: A modern urologic dilemma. Eur Urol. 2007;52(3):639-641.

8. Scattoni V, Zlotta A, Montironi R, Schulman C, Rigatti P, Montorsi F. Extended and saturation prostatic biopsy in the diagnosis and characterisation of prostate cancer: a critical analysis of the literature. Eur Urol. 2007;52(5):1309-1322.

9. Fleshner N, Klotz L. Role of "saturation biopsy" in the detection of pros tate cancer among difficult diagnostic cases. Urology. 2002;60(1):93-97.

10. Djavan B, Ravery V, Zlotta A, Dobronski P, Dobrovits M, Fakhari M, et al. Prospective evaluation of prostate cancer detected on biopsies 1, 2, 3 and 4: When should we stop?. J Urol. 2001;166(5):1679-1683.

11. Van Renterghem K, Van Koeveringe G, Achten R, Van Kerrebroeck P. Clinical relevance of transurethral resection of the prostate in "asymptomatic" patients with an elevated prostate-specific antigen level. Eur Urol. 2007;52(3):819-826.

12. Steuber T, Karakiewicz PI, Augustin H, Erbersdobler A, Lange I, Haese A, et al. Transition zone cancers undermine the predictive accuracy of Partin table stage predictions. J Urol. 2005;173(3):737-741.

13. Djavan B, Remzi M, Zlotta AR, Ravery V, Hammerer P, Reissigl A, et al Complexed prostate-specific antigen, complexed prostate-specific antigen density of total and transition zone, complexed/total prostate-specific antigen ratio, free-to-total prostate-specific antigen ratio, density of tota and transition zone prostate-specific antigen: Results of the Prospective Multicenter European Trial. Urology. 2002;60(4 Suppl 1):4-9.

14. Ornstein DK, Rao GS, Smith DS, Andriole GL. The impact of systematic prostate biopsy on prostate cancer incidence in men with symptomatic benign prostatic hyperplasia undergoing transurethral resection of the prostate. J Urol. 1997; 157(3):880-884.

15. Pelzer AE, Bektic J, Berger AP, Halpern EJ, Koppelstätter F, Klauser A, et al. Are transition zone biopsies still necessary to improve prostate cancer detection? Results from the Tyrol Screening project. Eur Urol. 2005;48(6):916-921.

16. Kirkham APS, Emberton M, Allen C. How good is MRI in detecting and characterising cancer within the prostate?. Eur Urol. 2006;50(6):1163-1175.

17. Amsellem-Ouazana D, Younes P, Conquy S, Peyromaure M, Flam T, Debré B, et al. Negative prostatic biopsies in patients with a high risk of prostate cancer. Is the combination of endorectal MRI and magnetic resonance spectroscopy imaging (MRSI) a useful tool? A preliminary study. Eur Urol. 2005;47(5):582-586.

18. Niesel T, Breul J, Hartung R. Diagnostic value of additional systematic prostate biopsies in patients undergoing transurethral resection of the prostate. Urology. 1997;49(6): 869-874

19. Djavan B, Remzi M, Marberger M. When to biopsy and when to stop biopsying. Urol Clin North Am. 2003;30(2):253-262.

20. Heidenreich A, Aus G, Bolla M, Joniau S, Matveev VB, Schmid HP, et al EAU guidelines on prostate cancer. Eur Urol. 2008;53(1):68-80.

21. Wendt-Nordahl G, Häcker A, Reich O, Djavan B, Alken P, Michel MS The Vista system: a new bipolar resection device for endourological procedures: comparison with conventional resectoscope. Eur Urol 2004;46(5):586-590.

Correspondencia autor: Dr. A. Rodríguez Alonso

Servicio de Urología

Hospital Arquitecto Marcide - Profesor Novoa Santos

Ctra. San Pedro de Leixa s/n - 15405 Ferrol, La Coruña

Tel.: 981334052

E-mail autor: ara68@orange.es

Información artículo: Original - Cáncer de próstata

Trabajo recibido: febrero 2008

Trabajo aceptado: marzo 2008 